\title{
Ti-24Nb-4Zr-8Sn Alloy Pedicle Screw Improves Internal Vertebral Fixation by Reducing Stress-Shielding Effects in a Porcine Model
}

\author{
Yang Qu, Shuang Zheng, Rongpeng Dong, Mingyang Kang, Haohan Zhou, \\ Dezhi Zhao, and Jianwu Zhao (iD)
}

Department of Orthopedics, The Second Hospital of Jilin University, Changchun 130041, China

Correspondence should be addressed to Jianwu Zhao; zhao20130922@163.com

Received 4 October 2017; Revised 9 January 2018; Accepted 11 January 2018; Published 8 February 2018

Academic Editor: Weijie Fu

Copyright (C) 2018 Yang Qu et al. This is an open access article distributed under the Creative Commons Attribution License, which permits unrestricted use, distribution, and reproduction in any medium, provided the original work is properly cited.

\begin{abstract}
To ensure the biomechanical properties of Ti-24Nb-4Zr-8Sn, stress-shielding effects were compared between Ti-24Nb-4Zr- $8 \mathrm{Sn}$ and Ti-6Al-4V fixation by using a porcine model. Twelve thoracolumbar spines (T12-L5) of 12-month-old male pigs were randomly divided into two groups: Ti-24Nb-4Zr-8Sn (EG, $n=6$ ) and Ti-6Al-4V (RG, $n=6$ ) fixation. Pedicle screw was fixed at the outer edge of L4-5 vertebral holes. Fourteen measuring points were selected on the front of transverse process and middle and posterior of L4-5 vertebra. Electronic universal testing machine was used to measure the strain resistance of measuring points after forward and backward flexion loading of $150 \mathrm{~N}$. Meanwhile, stress resistance was compared between both groups. The strain and stress resistance of measurement points $1,2,5,6,9$, and $10-14$ in Ti-24Nb-4Zr-8Sn fixation was lower than that of Ti-6Al-4V fixation after forward and backward flexion loading $(P<0.05)$. The strain and stress resistance of measurement points $3,4,7$, and 8 was higher in Ti-24Nb$4 \mathrm{Zr}-8 \mathrm{Sn}$ fixation than that of Ti-6Al-4V fixation $(P<0.05)$. Stress-shielding effects of Ti-24Nb-4Zr-8Sn internal fixation were less than that of Ti-6Al-4V internal fixation. These results suggest that Ti-24Nb-4Zr-8Sn elastic fixation has more biomechanical goals than conventional Ti-6Al-4V internal fixation by reducing stress-shielding effects.
\end{abstract}

\section{Introduction}

With global population aging, the incidence of degenerative lumbar degeneration has increased [1]. Vertebral fusion has become a gold standard for the treatment of lumbar degenerative diseases in spine surgeons [2,3]. Lumbar fusion of clinical application has been significantly improved in past decades, and rigid internal fixation technology has increased lumbar fusion rates $[4,5]$.

Low back pain induced by degenerative diseases is often experienced by the individuals beyond middle age. The standard treatment is decompression and interbody fusion, which is followed by a pedicle screw-rod system to stabilize spine $[6,7]$. Conventional pedicle screw-rod system is made from the titanium alloy Ti-6A1- $4 \mathrm{~V}$, which is biocompatible, corrosion resistant, bendable, and stiff enough to provide sufficient stability for spine [8]. However, the elastic modulus of Ti-6Al-4V (110 GPa) is much higher than that of cortical bone (18 GPa), and thus it changes spinal kinetics. After the implantation of Ti-6A1-4V, the motion of adjacent segments increases to compensate the reduced motion of implanted segments, which accelerate adjacent disc degeneration [9]. A stress-shielding effect, in which some stresses on vertebral bodies are shifted to pedicle-rod systems, decelerates intervertebral fusion and increases the looseness of pedicle screw. To eliminate these complications, some dynamic fixation systems have been developed. The most used one is the Dynesys Dynamic Stabilization System [10, 11], in which titanium alloy rods are replaced with polymolecular cords and spacers. However, the long-term clinical outcomes are not much better than expected [12].

Furthermore, due to strong stress-shielding effects caused by internal fixation, bone stress stimulation was significantly reduced, leading to bone reconstruction and absorption [13]. Biomechanical measurement of fusion techniques shows that all fusion will increase the stress of adjacent vertebrae [14]. Animal experiments also confirmed that strong internal fixation could cause stress occlusion and osteoporosis [15]. 
The increase of the stress near the segment after strong internal fixation accelerates the degeneration of adjacent segment disc $[16,17]$, which has become an urgent problem that needs to be solved after lumbar fusion.

In order to solve the problem caused by strong internal fixation, the researchers induce the concept of elastic fixation of spine and the premise of skeletal stability [18]. To reduce the load of the internal fixation and increase sharing load of spine, it is necessary to reduce the stress block and focus. At present, most researchers have applied intramedullary nailing or bone plate, joint replacement prosthesis implantation, and postoperative fixation of spine after internal fixation $[19,20]$. However, the degree of occlusion remains unclear [21, 22]. The data show that there is no significant difference between the elastic internal and rigid internal fixation system in the therapy of lumbar degenerative lesions [23]. Low modulus beta-titanium alloys are hoped to provide good biocompatibility and alleviate stress-shielding effects. Titanium alloy Ti$6 \mathrm{Al}-4 \mathrm{~V}$ is widely used for implants, which are characterized by high elastic modulus that may induce undesirable stressshielding effects and toxicity [24]. Ti-24Nb-4Zr-8Sn alloy, as a low modulus $(49 \mathrm{GPa})$, has been approved to a potential bone load-bearing implant material without toxicity [25].

However, the effects of Ti-24Nb-4Zr-8Sn and Ti-6Al$4 \mathrm{~V}$ fixation on shielding stress were seldom compared. To ensure biomechanical properties of Ti-24Nb-4Zr-8Sn, this experiment is performed to compare the effects of $\mathrm{Ti}-24 \mathrm{Nb}$ 4Zr-8Sn and Ti-6Al-4V fixation on shielding stress. Before and after internal fixation, the strain and stress resistance values of the two kinds of internal fixation on vertebra was compared under the same flexion load.

\section{Materials and Methods}

2.1. Materials. Twelve thoracolumbar spines (T12-L5) were obtained from 12-month male pigs. Before obtaining the specimens, all spines had no damage and deformity after being scanned by X-ray. All vertebra were randomly assigned into elastic $\mathrm{Ti}-24 \mathrm{Nb}-4 \mathrm{Zr}-8 \mathrm{Sn}$ (EG, $n=6$ ) and rigid $\mathrm{Ti}-$ $6 \mathrm{Al}-4 \mathrm{~V}(\mathrm{RG}, n=6)$ fixation groups. Scalpel, posterior longitudinal ligament, anterior longitudinal ligament, and facet joint were removed by scalpel and spatula (Figure 1(a)). The specimens were numbered and basic dimensions were measured (Figures 1(b)-1(d)). Lumbar spine specimens were embedded and fixed with denture base resin solution. Figure 1(e) showed the embedded species. Strain measurement was performed in both groups. The resistance of strain gauge was $121.4 \pm 0.1$ and gauge sensitivity coefficient was 2.14. Two kinds of pedicle screws Ti-24Nb-4Zr-8Sn and Ti-6Al$4 \mathrm{~V}$ were used to fix vertebra as Figures $1(\mathrm{f})$ and $1(\mathrm{~g})$ showed.

2.2. The Establishment of Measuring Points. With a finite element (FE) model and lumbar kinematic analysis according to earlier reports [26-28], biomechanical parameters with flexion loading were predicted. The geometry used in FE model of L4-5 was reconstructed across a porcine thoracolumbar spine. Before and after fixation at L4-5, the maximum strain and stress points were predicted after forward and backward flexion loading.
A total of 14 measuring points with possible high strain and stress resistance were predicted with a finite element (FE) model and lumbar kinematic analysis. Measuring points numbers 1 and 2 were found at left faces of L4-5 vertebra (Figure 2(a)). Measuring points numbers 3, 4, and 7-10 were found at middle faces of L4-5 vertebra (Figure 2(b)). Measuring points 5 and 6 were found at right faces of L45 vertebra (Figure 2(c)). Measuring points 11-14 were found at the posterior of L4-5 vertebra (Figure 2(d)). Figure 2(e) showed the measuring points used in Ti-24Nb-4Zr-8Sn group and Figure 2(f) showed measuring points used in $\mathrm{Ti}-$ 6A1-4V group.

\subsection{Measurement of Strain Resistance after Internal Fixation} and Flexion Load. The load was transmitted by the load cell with $300 \mathrm{~N}$ ranges, and the displacement was transmitted by photoelectric encoder. First of all, each specimen was installed in the test machine. Each specimen was loaded and unloaded 20 times for preconditioning processing. The strain gauge wire of each measuring point of the specimen was connected with the bridge arm of the dynamic resistance strain gauge junction box and bridged in a half way. The temperature compensation was adapted to the external condition. A load of $150 \mathrm{~N}$ was applied to the specimen at a load with increasing rate of $2 \mathrm{~mm} / \mathrm{min}$ (the load applied in this experiment was within the physiological load range). The strain resistance of each measuring point was measured by dynamic resistance strain gauge. After forward and backward flexion load, strain resistance was measured before pedicle screw fixation (Figures 3(a) and 3(b)). After forward flexion load, strain resistance was measured in Ti-24Nb-4Zr-8Sn group (Figure 3(c)) and Ti-6A1-4V group (Figure 3(d)) after pedicle screw fixation. Strain resistance was also measured in. After backward flexion load, strain resistance was measured in Ti-24Nb-4Zr-8Sn group (Figure 3(e)) and Ti-6A1-4V group (Figure 3(f)) after pedicle screw fixation.

\subsection{Each Group of Specimens of Pedicle Screw Fixation after} Internal Fixation. As Figure 3 showed, pedicle screw was fixed before application of flexion load. Two kinds of pedicle screw were manufactured by medical instrument company (Chunlizhengda Co., Beijing, China) by using titanium alloy Ti-24Nb-4Zr-8Sn (49 GPa) or Ti-6A1-4V (110 GPa). The lumbar posterior (L4, L5) was fixed with Ti-24Nb-4Zr-8Sn fixator or Ti-6A1-4V fixator. Ten points for pedicle screw fixation were determined. In order to measure the internal rod fixation and the junction of the strain, four additional points were selected for the internal fixation and pedicle screw at the junction (11-14 points). The measurement points were shown in Figure 2. The measuring points were the same between the EG and the RG groups (Figures 2(e) and 2(f)).

For the measurement of the strain gauge after pedicle screw fixation: the same model, batch, sensitivity coefficient, and resistance of strain gauge were used. The stress measurement was performed to measure the strain of the rats under the flexion load before the internal fixation of pedicle screw under flexion load. Ti-24Nb-4Zr-8Sn and Ti-6A1-4V fixation were performed under 150-N flexion load shown in Figures $2(\mathrm{c})-2(\mathrm{f})$. 


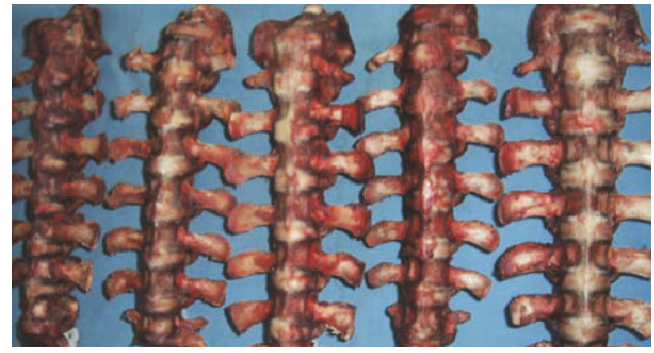

(a)

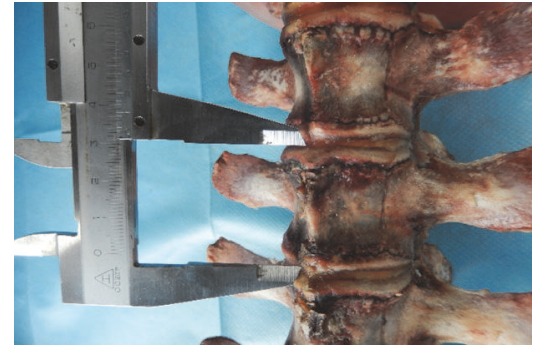

(b)

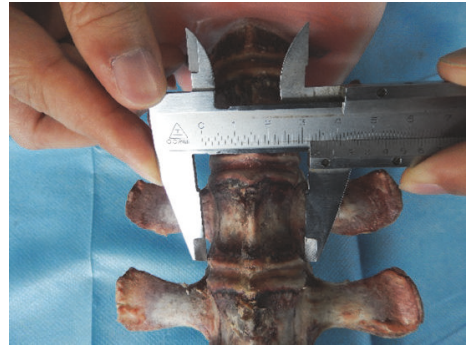

(c)

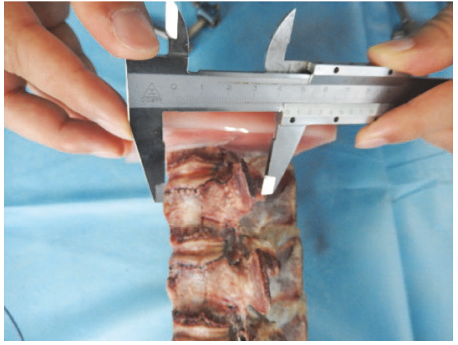

(d)

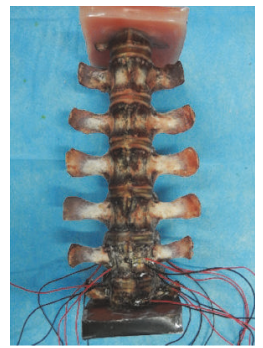

(e)

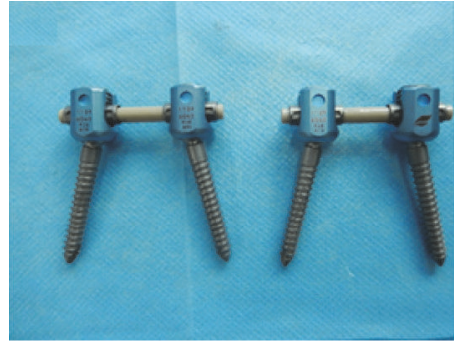

(f)

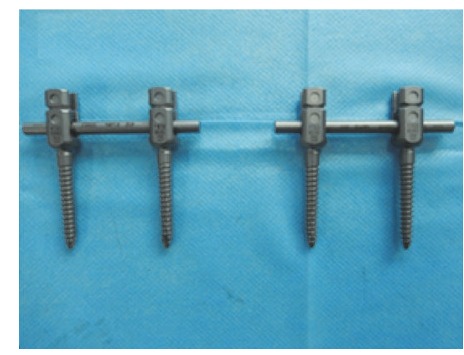

(g)

FIGURE 1: Specimen preparation. (a) Pig spine specimens with removal of muscle and other soft tissues. (b) Measurement of vertebral height. (c) Measurement of vertebral width. (d) Measurement of pedicle screw channel length. (e) Embedded specimen after fixation. (f) Ti-24Nb4Zr-8Sn pedicle screw. (g) Ti-6Al-4V pedicle screw.

2.5. Stress Calculation. The stress formula is provided as follows:

$$
\sigma=E \varepsilon,
$$

where $\sigma$ is the stress, $\varepsilon$ is the strain, and $E$ is the elastic modulus. In this experiment, the modulus of rigid internal Ti-6Al-4V fixation system is $110 \mathrm{GPa}$, and the modulus of the Ti-24Nb-4Zr-8Sn elastic internal fixation system is about $70 \mathrm{GPa}$ [29], and the elastic modulus of the lumbar vertebrae is $19.2 \mathrm{GPa}$ [29].
2.6. Statistical Analysis Methods. SPSS software package 16.0 SPSS (Chicago, IL, USA) was used for data analysis. The difference between the two types of variable data of variance was analyzed by using Scheffe method and paired $t$-test. $P<$ 0.05 for the statistical difference was significant.

\section{Results}

3.1. The Strain Resistance Values of Measuring Points between Ti-24Nb-4Zr-8Sn Internal Fixations Were Similar with Ti6A1-4V before Internal Fixation. The strain resistance of Ti$24 \mathrm{Nb}-4 \mathrm{Zr}-8 \mathrm{Sn}$ fixation and Ti-6Al-4V fixation group was 


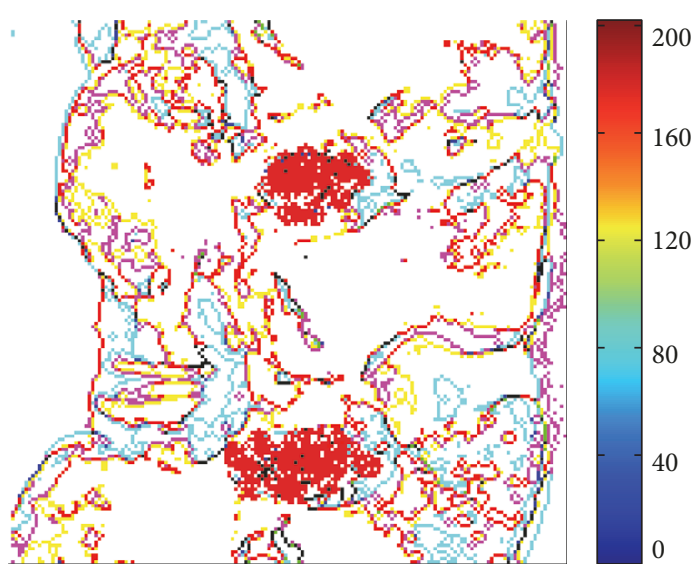

(a)

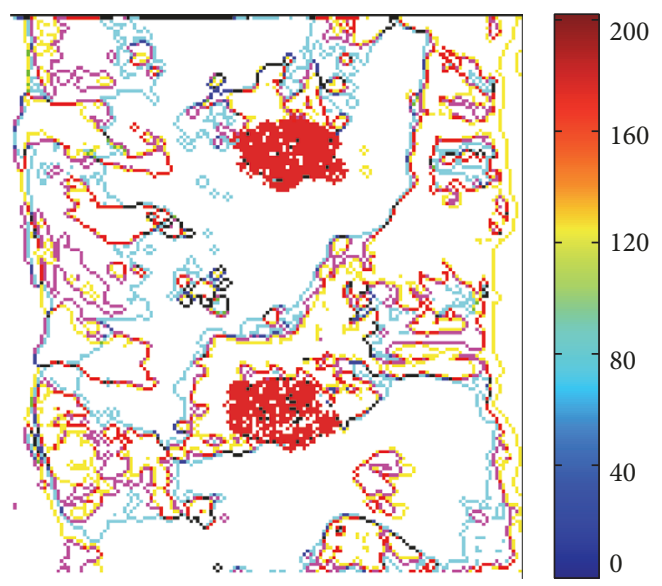

(c)

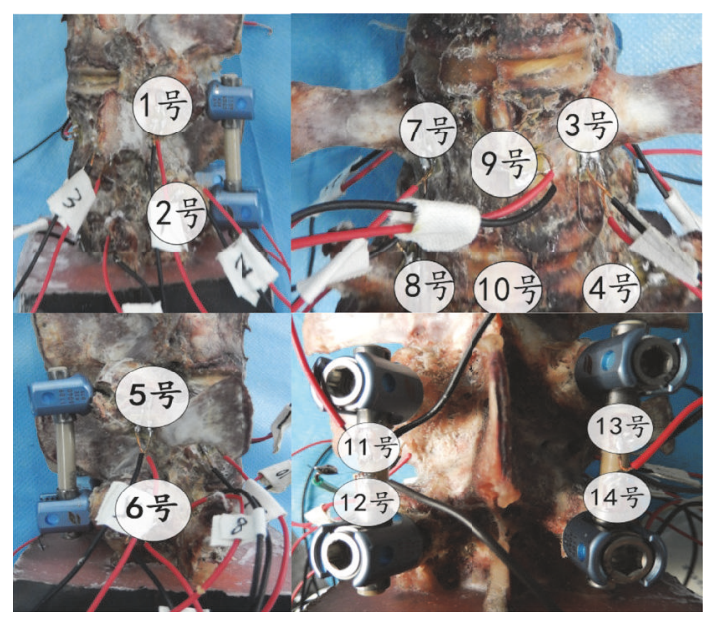

(e)

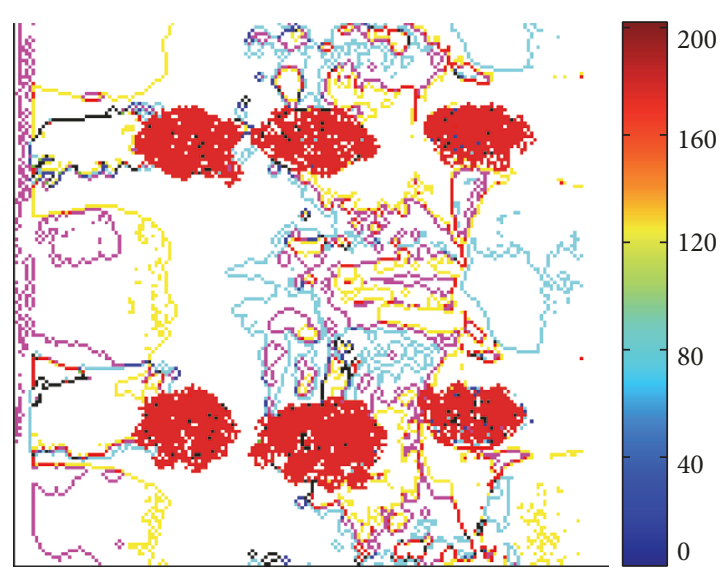

(b)

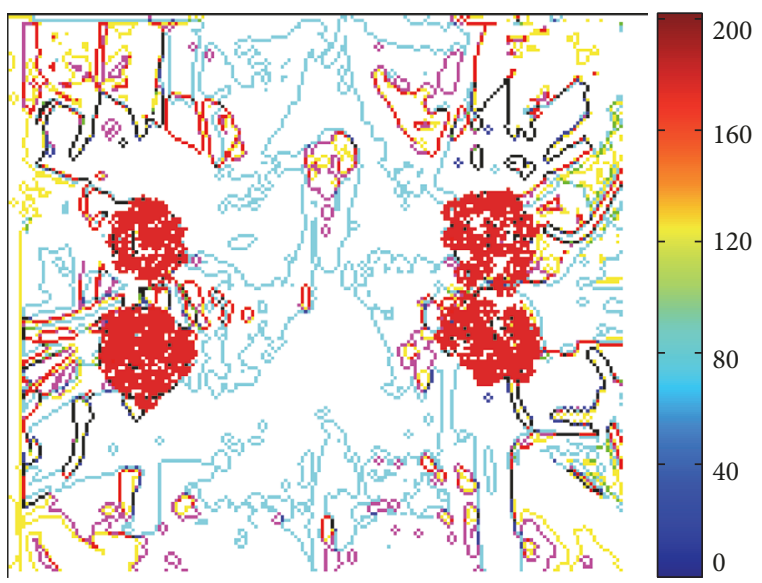

(d)

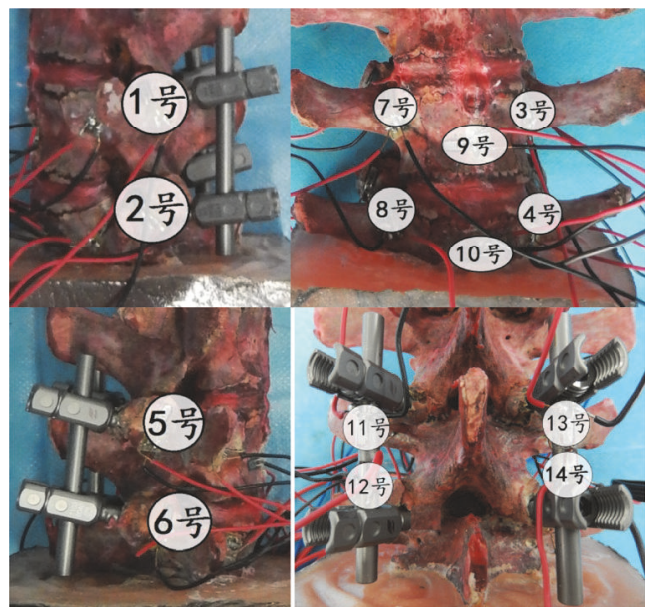

(f)

FIGURE 2: Measuring points predicted with finite element (FE) model and lumbar kinematic analysis. (a) Left L4-5 vertebra (measuring points numbers 1 and 2). (b) Middle L4-5 vertebra (measuring points numbers 3, 4, and 7-10). (c) Right L4-5 vertebra (measuring points 5 and 6 ). (d) Posterior L4-5 vertebra (measuring points 11-14). (e) Measuring points used in Ti-24Nb-4Zr-8Sn pedicle screw group. (f) Measuring points used in Ti-6A1-4V pedicle screw group. Number 1 point for left pedicle screw fixed at outside the hole of the lumbar vertebrae; number 2 point for left pedicle screw fixed at outside the hole of the lumbar vertebrae; number 3 points for pedicle screw fixed at left side of the transverse of waist L4 vertebral body. Number 5 point for pedicle screw fixed at outside the hole of vertebral vertebrae; number 6 points for right pedicle screw fixed below the ground of the lumbar vertebrae; number 7 points for right pedicle screw fixed at transverse sites of lumbar vertebrae; number 8 points for right pedicle screw fixed at transverse process; number 9 point for right pedicle screw fixed at front of waist L4 vertebral body; number 10 point for right pedicle screw fixed on the 10th vertebrae of the lumbar vertebrae. Number 11 point was at the junction of left fixation rod and L4 pedicle screw. Number 12 point was at the junction of left fixation rod and L5 pedicle screw. Number 13 point was at the junction of right fixation rod and L4 pedicle screw. Number 14 point was at the junction of right fixation rod and L5 pedicle screw. 


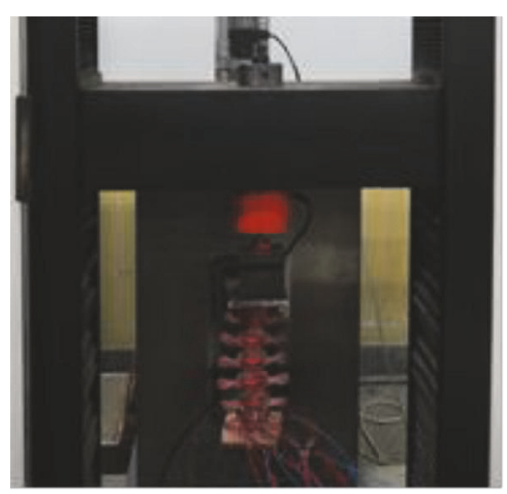

(a)

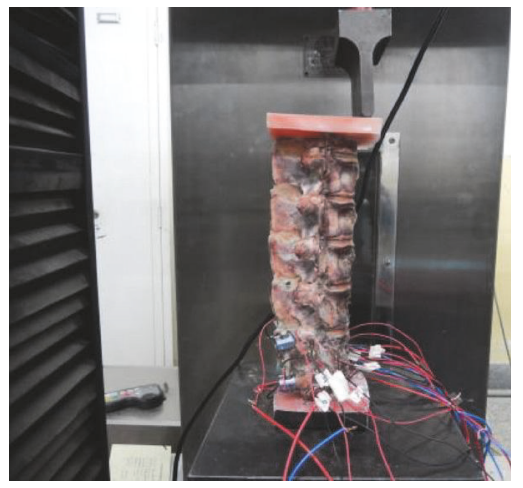

(c)

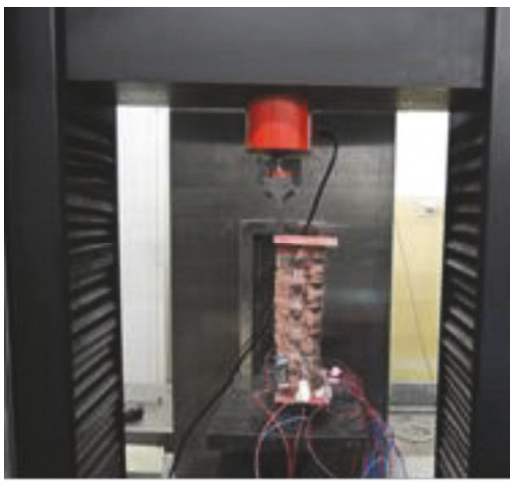

(e)

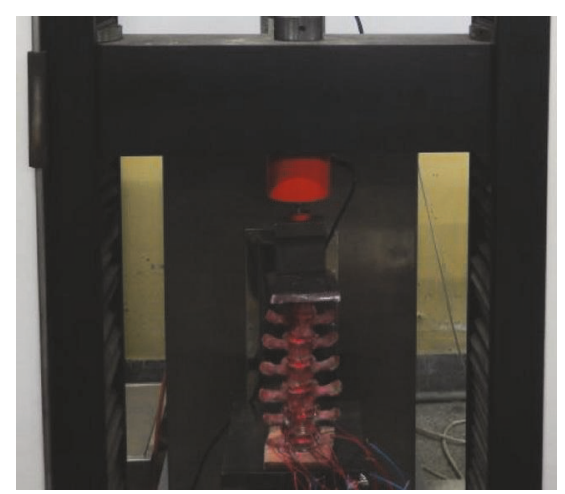

(b)

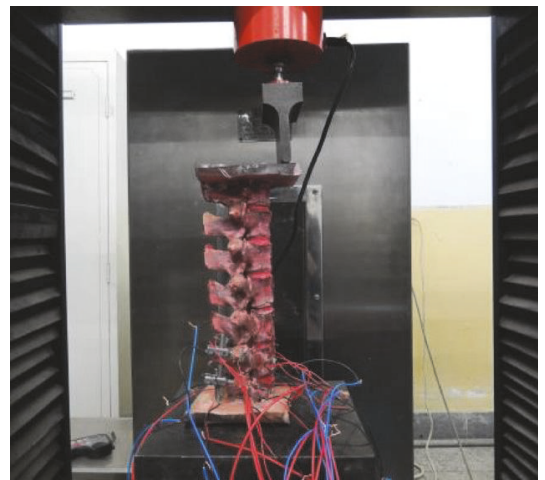

(d)

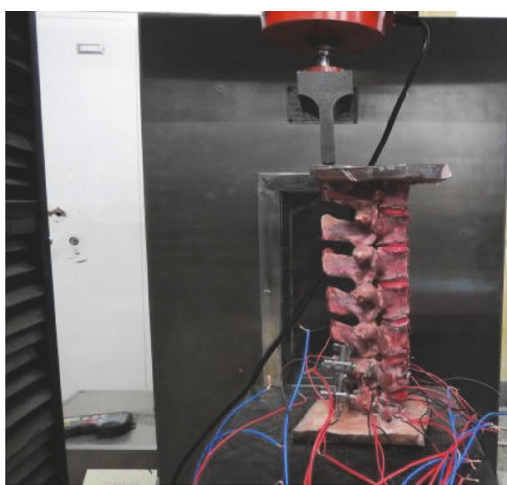

(f)

FIGURE 3: Strain resistance measurements. (a) After forward flexion load, strain resistance was measured before pedicle screw fixation. (b) After backward flexion load, strain resistance was measured before pedicle screw fixation. (c) After forward flexion load, strain resistance was measured in Ti-24Nb-4Zr-8Sn group after pedicle screw fixation. (d) After forward flexion load, strain resistance was measured in Ti-6A1-4V group after pedicle screw fixation. (e) After backward flexion load, strain resistance was measured in Ti-24Nb-4Zr-8Sn group after pedicle screw fixation. (f) After backward flexion load, strain resistance was measured in Ti-6Al-4V group after pedicle screw fixation.

compared after flexion loading. Before alloy fixation, there was no significantly statistical difference for strain resistance after forward flexion loading (Table 1) or backward flexion loading (Table 2) between the EG and the RG groups $(P>$ $0.05)$. The results suggested that the specimens were similar with the same strain resistance values of measuring points between the EG and the RG groups.

3.2. The Strain Resistance of Ti-24Nb-4Zr-8Sn Was Higher Than That of Ti-6A1-4V after Internal Fixation. Strain measurement showed that the strain resistance of the $\mathrm{Ti}$ $24 \mathrm{Nb}-4 \mathrm{Zr}-8 \mathrm{Sn}$ fixation group at $1,2,5,6$, 9, and 10-14 measuring was lower than that of the rigid internal fixation under forward flexion loading after fixation (Table $3, P<$ 0.05 ) and backward flexion loading (Table $4, P<0.05$ ). In contrast, strain resistance of $\mathrm{Ti}-24 \mathrm{Nb}-4 \mathrm{Zr}-8 \mathrm{Sn}$ elastic internal fixation at $3,4,7$, and 8 measuring points was higher than that of Ti-6A1-4V fixation under forward flexion loading after fixation (Table 3, $P<0.05$ ) and backward flexion loading (Table $4, P<0.05$ ). 
TABLE 1: The strain resistance of Ti-24Nb-4Zr-8Sn and Ti-6Al-4V fixation groups under forward flexion loading before fixation $\left(\mu \varepsilon, \times 10^{-6}\right)$.

\begin{tabular}{|c|c|c|c|c|c|c|c|}
\hline Measuring points & Sample 1 & Sample 2 & Sample 3 & Sample 4 & Sample 5 & Sample 6 & Mean \pm SD \\
\hline \multicolumn{8}{|l|}{1} \\
\hline EG & 130 & 131 & 133 & 127 & 124 & 129 & $129 \pm 3$ \\
\hline RG & 138 & 145 & 133 & 140 & 137 & 143 & $139 \pm 4$ \\
\hline \multicolumn{8}{|l|}{2} \\
\hline EG & 126 & 125 & 129 & 130 & 134 & 127 & $129 \pm 3$ \\
\hline RG & 127 & 134 & 139 & 136 & 129 & 135 & $133 \pm 5$ \\
\hline \multicolumn{8}{|l|}{3} \\
\hline EG & 155 & 148 & 159 & 161 & 155 & 150 & $155 \pm 5$ \\
\hline RG & 157 & 153 & 156 & 161 & 159 & 164 & $158 \pm 4$ \\
\hline \multicolumn{8}{|l|}{4} \\
\hline EG & 165 & 156 & 160 & 163 & 152 & 157 & $159 \pm 5$ \\
\hline RG & 165 & 159 & 163 & 157 & 160 & 159 & $161 \pm 3$ \\
\hline \multicolumn{8}{|l|}{5} \\
\hline EG & 128 & 123 & 127 & 131 & 130 & 129 & $128 \pm 3$ \\
\hline RG & 126 & 135 & 133 & 130 & 128 & 129 & $130 \pm 3$ \\
\hline \multicolumn{8}{|l|}{6} \\
\hline EG & 125 & 127 & 129 & 132 & 128 & 123 & $127 \pm 3$ \\
\hline $\mathrm{RG}$ & 129 & 126 & 134 & 133 & 125 & 127 & $129 \pm 4$ \\
\hline \multicolumn{8}{|l|}{7} \\
\hline EG & 161 & 168 & 164 & 158 & 167 & 159 & $163 \pm 4$ \\
\hline RG & 159 & 164 & 162 & 159 & 169 & 168 & $164 \pm 4$ \\
\hline \multicolumn{8}{|l|}{8} \\
\hline EG & 172 & 169 & 159 & 177 & 175 & 171 & $171 \pm 6$ \\
\hline RG & 170 & 165 & 170 & 157 & 165 & 172 & $167 \pm 5$ \\
\hline \multicolumn{8}{|l|}{9} \\
\hline EG & 188 & 195 & 193 & 189 & 191 & 192 & $191 \pm 3$ \\
\hline RG & 184 & 188 & 197 & 189 & 196 & 190 & $191 \pm 5$ \\
\hline \multicolumn{8}{|l|}{10} \\
\hline EG & 191 & 186 & 187 & 192 & 196 & 191 & $191 \pm 4$ \\
\hline $\mathrm{RG}$ & 187 & 190 & 186 & 195 & 188 & 193 & $190 \pm 4$ \\
\hline \multicolumn{8}{|l|}{11} \\
\hline EG & 192 & 189 & 202 & 193 & 188 & 197 & $194 \pm 5$ \\
\hline RG & 189 & 191 & 200 & 195 & 192 & 201 & $195 \pm 5$ \\
\hline \multicolumn{8}{|l|}{12} \\
\hline EG & 200 & 197 & 195 & 199 & 192 & 189 & $195 \pm 4$ \\
\hline RG & 196 & 188 & 190 & 200 & 196 & 196 & $194 \pm 4$ \\
\hline \multicolumn{8}{|l|}{13} \\
\hline EG & 188 & 194 & 192 & 197 & 201 & 196 & $195 \pm 4$ \\
\hline RG & 192 & 198 & 188 & 192 & 205 & 200 & $196 \pm 6$ \\
\hline \multicolumn{8}{|l|}{14} \\
\hline EG & 194 & 199 & 189 & 193 & 194 & 187 & $193 \pm 4$ \\
\hline RG & 189 & 195 & 192 & 183 & 187 & 195 & $190 \pm 5$ \\
\hline
\end{tabular}

3.3. Stress Resistance of Ti-24Nb-4Zr-8Sn Was Lower Than Ti6A1-4V after Internal Fixation. The stress resistance of $\mathrm{Ti}-$ $24 \mathrm{Nb}-4 \mathrm{Zr}-8 \mathrm{Sn}$ and $\mathrm{Ti}-6 \mathrm{Al}-4 \mathrm{~V}$ internal fixation after flexion load was compared. The results showed that stress resistance of Ti-24Nb-4Zr-8Sn fixation was lower than that of Ti-6A1$4 \mathrm{~V}$ fixation after forward flexion loading (Table 5, $\mathrm{P}<$ 0.05 ) and backward flexion loading (Table $6, P<0.05$ ) at measuring points $1,2,5,6,9$, and 10-14. In contrast, stress resistance of $\mathrm{Ti}-24 \mathrm{Nb}-4 \mathrm{Zr}-8 \mathrm{Sn}$ fixation was higher than that of Ti-6A1-4V fixation after forward flexion loading (Table 5, $P<0.05$ ) and backward flexion loading (Table $6, P<0.05$ ) at measuring points $3,4,7$, and 8 . All the results suggest that strain and stress resistance showed more stress-shielding effects in the RG group than in the EG group. 
TABLE 2: The strain resistance of Ti-24Nb-4Zr-8Sn and Ti-6A1-4V fixation groups under backward flexion loading before fixation $\left(\mu \varepsilon, \times 10^{-6}\right)$.

\begin{tabular}{|c|c|c|c|c|c|c|c|}
\hline Measuring points & Sample 1 & Sample 2 & Sample 3 & Sample 4 & Sample 5 & Sample 6 & Mean \pm SD \\
\hline \multicolumn{8}{|l|}{1} \\
\hline EG & -200 & -189 & -201 & -205 & -192 & -197 & $-197 \pm 6$ \\
\hline RG & -203 & -187 & -197 & -199 & -201 & -197 & $-197 \pm 6$ \\
\hline \multicolumn{8}{|l|}{2} \\
\hline EG & -205 & -196 & -198 & -192 & -193 & -201 & $-198 \pm 5$ \\
\hline RG & -204 & -196 & -193 & -201 & -195 & -203 & $-199 \pm 5$ \\
\hline \multicolumn{8}{|l|}{3} \\
\hline EG & -167 & -174 & -168 & -172 & -173 & -169 & $-171 \pm 3$ \\
\hline RG & -177 & -176 & -168 & -175 & -172 & -169 & $-173 \pm 4$ \\
\hline \multicolumn{8}{|l|}{4} \\
\hline EG & -178 & -169 & -177 & -169 & -174 & -170 & $-173 \pm 4$ \\
\hline RG & -173 & -169 & -175 & -174 & -178 & -165 & $-172 \pm 5$ \\
\hline \multicolumn{8}{|l|}{5} \\
\hline EG & -198 & -202 & -199 & -195 & -201 & -197 & $-199 \pm 3$ \\
\hline RG & -197 & -194 & -192 & -196 & -202 & -200 & $-197 \pm 4$ \\
\hline \multicolumn{8}{|l|}{6} \\
\hline EG & -207 & -209 & -196 & -203 & -208 & -195 & $-203 \pm 6$ \\
\hline $\mathrm{RG}$ & -204 & -202 & -197 & -193 & -196 & -199 & $-199 \pm 4$ \\
\hline \multicolumn{8}{|l|}{7} \\
\hline EG & -150 & -162 & -155 & -157 & -159 & -161 & $-157 \pm 4$ \\
\hline RG & -155 & -161 & -157 & -154 & -159 & -163 & $-158 \pm 3$ \\
\hline \multicolumn{8}{|l|}{8} \\
\hline EG & -167 & -178 & -168 & -172 & -170 & -169 & $-171 \pm 4$ \\
\hline RG & -173 & -175 & -166 & -161 & -167 & -169 & $-169 \pm 5$ \\
\hline \multicolumn{8}{|l|}{9} \\
\hline EG & -128 & -136 & -130 & -132 & -129 & -135 & $-132 \pm 3$ \\
\hline RG & -124 & -133 & -131 & -127 & -130 & -128 & $-129 \pm 3$ \\
\hline \multicolumn{8}{|l|}{10} \\
\hline EG & -134 & -137 & -133 & -137 & -135 & -130 & $-134 \pm 3$ \\
\hline $\mathrm{RG}$ & -132 & -130 & -137 & -135 & -129 & -131 & $-132 \pm 3$ \\
\hline \multicolumn{8}{|l|}{11} \\
\hline EG & -219 & -216 & -233 & -229 & -230 & -227 & $-226 \pm 7$ \\
\hline RG & -214 & -213 & -225 & -231 & -219 & -232 & $-222 \pm 8$ \\
\hline \multicolumn{8}{|l|}{12} \\
\hline EG & -231 & -227 & -218 & -231 & -237 & -226 & $-228 \pm 6$ \\
\hline RG & -228 & -231 & -221 & -242 & -234 & -220 & $-229 \pm 8$ \\
\hline \multicolumn{8}{|l|}{13} \\
\hline EG & -217 & -241 & -229 & -236 & -224 & -238 & $-231 \pm 9$ \\
\hline RG & -220 & -233 & -231 & -232 & -231 & -229 & $-229 \pm 5$ \\
\hline \multicolumn{8}{|l|}{14} \\
\hline EG & -224 & -223 & -236 & -240 & -229 & -237 & $-232 \pm 7$ \\
\hline RG & -233 & -220 & -231 & -236 & -238 & -241 & $-233 \pm 7$ \\
\hline
\end{tabular}

\section{Discussion}

The strain and stress resistance of the $\mathrm{Ti}-24 \mathrm{Nb}-4 \mathrm{Zr}-8 \mathrm{Sn}$ and Ti-6A1-4V fixation increased after flexion load, but the increase in $\mathrm{Ti}-6 \mathrm{Al}-4 \mathrm{~V}$ group was greater than that of $\mathrm{Ti}-24 \mathrm{Nb}-4 \mathrm{Zr}-8 \mathrm{Sn}$ group. Strain resistance of 10 measuring points in Ti-6A1-4V group was higher than that of Ti-24Nb4Zr-8Sn group (Tables 3 and $4, P<0.05$ ). Similarly, stress resistance of $\mathrm{Ti}-24 \mathrm{Nb}-4 \mathrm{Zr}-8 \mathrm{Sn}$ was also lower than that of Ti-6A1-4V group (Tables 5 and $6, P<0.05$ ). Normally, bone material with low modulus elasticity is less resistant to outside stress, while the material with high-modulus elasticity is more resistant to outside stress. The results showed that the stressshielding effects of Ti-24Nb-4Zr-8Sn fixation were small.

Stress conduction can be caused by lumbar degeneration [16], whereas back pain and other symptoms can 
TABLE 3: The strain resistance of $-24 \mathrm{Nb}-4 \mathrm{Zr}-8 \mathrm{Sn}$ and Ti-6Al-4V fixation groups under forward flexion loading after fixation $\left(\mu \varepsilon, \times 10^{-6}\right)$.

\begin{tabular}{|c|c|c|c|c|c|c|c|}
\hline Measuring points & Sample 1 & Sample 2 & Sample 3 & Sample 4 & Sample 5 & Sample 6 & Mean \pm SD \\
\hline \multicolumn{8}{|l|}{1} \\
\hline EG & 149 & 152 & 147 & 152 & 155 & 161 & $152.67 \pm 4.93^{*}$ \\
\hline RG & 188 & 179 & 187 & 193 & 196 & 189 & $188.66 \pm 5.82$ \\
\hline \multicolumn{8}{|l|}{2} \\
\hline EG & 155 & 160 & 153 & 145 & 162 & 154 & $154.83 \pm 5.98^{*}$ \\
\hline RG & 193 & 196 & 201 & 186 & 193 & 182 & $191.83 \pm 6.85$ \\
\hline \multicolumn{8}{|l|}{3} \\
\hline EG & 134 & 131 & 129 & 133 & 126 & 130 & $130.50 \pm 2.88^{*}$ \\
\hline RG & 105 & 97 & 99 & 102 & 106 & 98 & $101.17 \pm 3.76$ \\
\hline \multicolumn{8}{|l|}{4} \\
\hline EG & 137 & 126 & 124 & 135 & 129 & 135 & $131.00 \pm 5.40^{*}$ \\
\hline RG & 107 & 103 & 104 & 99 & 103 & 101 & $102.83 \pm 2.71$ \\
\hline \multicolumn{8}{|l|}{5} \\
\hline EG & 156 & 147 & 149 & 153 & 160 & 156 & $153.50 \pm 4.85^{*}$ \\
\hline RG & 203 & 192 & 183 & 179 & 197 & 185 & $189.83 \pm 9.13$ \\
\hline \multicolumn{8}{|l|}{6} \\
\hline EG & 152 & 154 & 148 & 157 & 155 & 163 & $154.83 \pm 5.04^{*}$ \\
\hline RG & 189 & 198 & 195 & 184 & 204 & 197 & $194.50 \pm 7.06$ \\
\hline \multicolumn{8}{|l|}{7} \\
\hline EG & 132 & 125 & 129 & 136 & 127 & 133 & $130.33 \pm 4.08^{*}$ \\
\hline RG & 96 & 103 & 105 & 102 & 98 & 96 & $100.00 \pm 3.85$ \\
\hline \multicolumn{8}{|l|}{8} \\
\hline EG & 134 & 129 & 124 & 131 & 135 & 137 & $131.67 \pm 4.72^{*}$ \\
\hline RG & 99 & 97 & 108 & 107 & 106 & 103 & $103.33 \pm 4.50$ \\
\hline \multicolumn{8}{|l|}{9} \\
\hline EG & 129 & 135 & 127 & 132 & 131 & 135 & $131.50 \pm 3.21^{*}$ \\
\hline RG & 159 & 165 & 161 & 157 & 163 & 162 & $161.17 \pm 2.86$ \\
\hline \multicolumn{8}{|l|}{10} \\
\hline EG & 132 & 130 & 128 & 135 & 134 & 129 & $131.33 \pm 2.80^{*}$ \\
\hline RG & 164 & 157 & 163 & 160 & 159 & 166 & $161.50 \pm 3.39$ \\
\hline \multicolumn{8}{|l|}{11} \\
\hline EG & 119 & 122 & 125 & 117 & 127 & 133 & $123.83 \pm 5.81^{*}$ \\
\hline RG & 152 & 149 & 162 & 153 & 148 & 157 & $153.50 \pm 5.24$ \\
\hline \multicolumn{8}{|l|}{12} \\
\hline EG & 126 & 122 & 132 & 124 & 131 & 118 & $125.50 \pm 5.36^{*}$ \\
\hline RG & 160 & 157 & 155 & 159 & 152 & 149 & $155.33 \pm 4.23$ \\
\hline \multicolumn{8}{|l|}{13} \\
\hline EG & 125 & 124 & 127 & 123 & 118 & 124 & $123.50 \pm 302^{*}$ \\
\hline RG & 148 & 154 & 152 & 157 & 161 & 156 & $154.67 \pm 4.48$ \\
\hline \multicolumn{8}{|l|}{14} \\
\hline EG & 129 & 116 & 119 & 129 & 125 & 118 & $122.67 \pm 5.75^{*}$ \\
\hline RG & 154 & 159 & 149 & 153 & 154 & 147 & $152.67 \pm 4.23$ \\
\hline
\end{tabular}

Note. ${ }^{*} P<0.05$ versus an RG group. Strain values are positive (tensile) due to elongation caused by forward flexion load.

be reduced or even disappear if lumbar dynamic internal fixation system precisely limits the activities of the vertebral body [30]. Theoretically, lumbar dynamic stability system reduces adjacent segment degeneration and the stress on the intervertebral bone graft and promotes bone healing and spine recovery. However, high-level stress will cause spinal cord compression and induce nerve tissue damage
[31]. Lumbar Ti-24Nb-4Zr-8Sn fixation system is a dynamic stability system made of titanium alloy and polyether ether ketone (PEEK) materials [32]. The materials are safe for patient health and have high strength, excellent mechanical properties, good self-lubricating, corrosion resistance, wear, and other characteristics [33]. Its ultralow elastic modulus in a simple bar-shaped design can achieve a satisfactory 
TABLE 4: The strain resistance of Ti-24Nb-4Zr-8Sn and Ti-6Al-4V fixation groups under backward flexion loading after fixation $\left(\mu \varepsilon, \times 10^{-6}\right)$.

\begin{tabular}{|c|c|c|c|c|c|c|c|}
\hline Measuring points & Sample 1 & Sample 2 & Sample 3 & Sample 4 & Sample 5 & Sample 6 & Mean \pm SD \\
\hline \multicolumn{8}{|l|}{1} \\
\hline EG & -245 & -254 & -249 & -257 & -261 & -258 & $-254 \pm 6^{*}$ \\
\hline RG & -310 & -305 & -319 & -304 & -318 & -301 & $-310 \pm 8$ \\
\hline \multicolumn{8}{|l|}{2} \\
\hline EG & -251 & -260 & -257 & -249 & -255 & -253 & $-254 \pm 4^{*}$ \\
\hline RG & -321 & -317 & -306 & -320 & -309 & -305 & $-313 \pm 7$ \\
\hline \multicolumn{8}{|l|}{3} \\
\hline EG & -109 & -127 & -130 & -121 & -129 & -123 & $-123 \pm 8^{*}$ \\
\hline RG & -92 & -96 & -89 & -90 & -88 & -91 & $-91 \pm 3$ \\
\hline \multicolumn{8}{|l|}{4} \\
\hline EG & -121 & -123 & -107 & -114 & -125 & -110 & $-117 \pm 7^{*}$ \\
\hline RG & -87 & -98 & -85 & -92 & -96 & -93 & $-92 \pm 5$ \\
\hline \multicolumn{8}{|l|}{5} \\
\hline EG & -260 & -254 & -257 & -249 & -262 & -255 & $-256 \pm 5^{*}$ \\
\hline RG & -314 & -321 & -317 & -305 & -321 & -318 & $-316 \pm 6$ \\
\hline \multicolumn{8}{|l|}{6} \\
\hline EG & -257 & -248 & -263 & -246 & -250 & -259 & $-254 \pm 7^{*}$ \\
\hline RG & -309 & -307 & -312 & -318 & -317 & -311 & $-312 \pm 4$ \\
\hline \multicolumn{8}{|l|}{7} \\
\hline EG & -124 & -114 & -131 & -109 & -123 & -122 & $-121 \pm 8^{*}$ \\
\hline RG & -87 & -84 & -95 & -97 & -88 & -93 & $-91 \pm 5$ \\
\hline \multicolumn{8}{|l|}{8} \\
\hline EG & -127 & -109 & -128 & -117 & -125 & -120 & $-121 \pm 7^{*}$ \\
\hline RG & -94 & -89 & -90 & -93 & -97 & -85 & $-91 \pm 4$ \\
\hline \multicolumn{8}{|l|}{9} \\
\hline EG & -87 & -83 & -91 & -82 & -88 & -90 & $-87 \pm 4^{*}$ \\
\hline RG & -98 & -117 & -104 & -117 & -103 & -119 & $-110 \pm 9$ \\
\hline \multicolumn{8}{|l|}{10} \\
\hline EG & -82 & -90 & -87 & -84 & -81 & -89 & $-86 \pm 4^{*}$ \\
\hline RG & -105 & -110 & -109 & -121 & -105 & -114 & $-111 \pm 6$ \\
\hline \multicolumn{8}{|l|}{11} \\
\hline EG & -145 & -151 & -155 & -150 & -163 & -158 & $-154 \pm 6^{*}$ \\
\hline RG & -199 & -196 & -213 & -209 & -210 & -207 & $-206 \pm 7$ \\
\hline \multicolumn{8}{|l|}{12} \\
\hline EG & -156 & -148 & -161 & -157 & -153 & -148 & $-154 \pm 5^{*}$ \\
\hline RG & -211 & -207 & -198 & -211 & -217 & -206 & $-208 \pm 6$ \\
\hline \multicolumn{8}{|l|}{13} \\
\hline EG & -153 & -147 & -162 & -147 & -165 & -154 & $-155 \pm 8^{*}$ \\
\hline RG & -197 & -221 & -209 & -216 & -204 & -218 & $-211 \pm 9$ \\
\hline \multicolumn{8}{|l|}{14} \\
\hline EG & -160 & -155 & -153 & -156 & -161 & -146 & $-155 \pm 5^{*}$ \\
\hline RG & -204 & -203 & -216 & -220 & -209 & -217 & $-212 \pm 7$ \\
\hline
\end{tabular}

Note. ${ }^{*} P<0.05$ versus an RG group. Strain values are negative due to compressive pressure caused by backward flexion load.

dynamic fixation effect. Ti-24Nb-4Zr-8Sn fixation system can reduce the stress resistance of titanium pedicle screw when the stress resistance acts on the elastic connecting rod with certain microaction because polyethylene fiber elastic connecting rod instead of rigid connecting rod with titanium alloys is used. The system reduces vertebral osteoporosis and pedicle screw loosening, fracture, and other risks and maintains intervertebral space and active function.

The load of the segment slows down the degeneration of adjacent segment discs. After the internal fixation of the spine, biomechanical properties of the column are restored, the load sharing of the internal fixation system is reduced, and the reduction of internal fixation load is important 
TABLE 5: The stress resistance of Ti-24Nb-4Zr-8Sn and Ti-6Al-4V fixation groups under forward flexion loading after fixation (MPa).

\begin{tabular}{|c|c|c|c|c|c|c|c|}
\hline Measuring points & Sample 1 & Sample 2 & Sample 3 & Sample 4 & Sample 5 & Sample 6 & Mean \pm S.D \\
\hline \multicolumn{8}{|l|}{1} \\
\hline EG & 2.86 & 2.92 & 2.82 & 2.92 & 2.98 & 3.09 & $2.93 \pm 0.09^{*}$ \\
\hline RG & 3.61 & 3.44 & 3.59 & 3.71 & 3.76 & 3.63 & $3.62 \pm 0.11$ \\
\hline \multicolumn{8}{|l|}{2} \\
\hline EG & 2.98 & 3.07 & 2.94 & 2.78 & 3.11 & 2.96 & $2.97 \pm 0.12^{*}$ \\
\hline RG & 3.71 & 3.76 & 3.86 & 3.57 & 3.71 & 3.49 & $3.68 \pm 0.13$ \\
\hline \multicolumn{8}{|l|}{3} \\
\hline EG & 2.57 & 2.52 & 2.48 & 2.55 & 2.42 & 2.5 & $2.51 \pm 0.05^{*}$ \\
\hline RG & 2.02 & 1.86 & 1.9 & 1.96 & 2.04 & 1.88 & $1.94 \pm 0.08$ \\
\hline \multicolumn{8}{|l|}{4} \\
\hline EG & 2.63 & 2.42 & 2.38 & 2.59 & 2.48 & 2.59 & $2.52 \pm 0.10^{*}$ \\
\hline RG & 2.05 & 1.98 & 2 & 1.9 & 1.98 & 1.94 & $1.98 \pm 0.05$ \\
\hline \multicolumn{8}{|l|}{5} \\
\hline EG & 3 & 2.82 & 2.86 & 2.94 & 3.07 & 3 & $2.95 \pm 0.09^{*}$ \\
\hline RG & 3.9 & 3.69 & 3.51 & 3.44 & 3.78 & 3.55 & $3.65 \pm 0.18$ \\
\hline \multicolumn{8}{|l|}{6} \\
\hline EG & 2.92 & 2.96 & 2.84 & 3.01 & 2.98 & 3.13 & $2.97 \pm 0.10^{*}$ \\
\hline RG & 3.63 & 3.8 & 3.74 & 3.53 & 3.92 & 3.78 & $3.73 \pm 0.14$ \\
\hline \multicolumn{8}{|l|}{7} \\
\hline EG & 2.53 & 2.4 & 2.48 & 2.61 & 2.44 & 2.55 & $2.50 \pm 0.08^{*}$ \\
\hline RG & 1.84 & 1.98 & 2.02 & 1.96 & 1.88 & 1.84 & $1.92 \pm 0.08$ \\
\hline \multicolumn{8}{|l|}{8} \\
\hline EG & 2.57 & 2.48 & 2.38 & 2.52 & 2.59 & 2.63 & $2.53 \pm 0.09^{*}$ \\
\hline RG & 1.9 & 1.86 & 2.07 & 2.05 & 2.04 & 1.98 & $1.98 \pm 0.09$ \\
\hline \multicolumn{8}{|l|}{9} \\
\hline EG & 2.48 & 2.59 & 2.44 & 2.53 & 2.52 & 2.59 & $2.53 \pm 0.06^{*}$ \\
\hline RG & 3.05 & 3.17 & 3.09 & 3.01 & 3.13 & 3.11 & $3.09 \pm 0.05$ \\
\hline \multicolumn{8}{|l|}{10} \\
\hline EG & 2.53 & 2.5 & 2.46 & 2.59 & 2.57 & 2.48 & $2.52 \pm 0.05^{*}$ \\
\hline RG & 3.15 & 3.01 & 3.13 & 3.07 & 3.05 & 3.19 & $3.01 \pm 0.06$ \\
\hline \multicolumn{8}{|l|}{11} \\
\hline EG & 8.97 & 8.79 & 9.56 & 9.03 & 8.73 & 9.26 & $9.06 \pm 0.31^{*}$ \\
\hline RG & 13.09 & 13.42 & 13.75 & 12.87 & 13.97 & 14.63 & $13.62 \pm 0.64$ \\
\hline \multicolumn{8}{|l|}{12} \\
\hline EG & 9.44 & 9.26 & 9.15 & 9.38 & 8.97 & 8.79 & $9.17 \pm 0.25^{*}$ \\
\hline RG & 13.86 & 13.42 & 14.52 & 13.64 & 14.41 & 12.98 & $13.80 \pm 0.59$ \\
\hline \multicolumn{8}{|l|}{13} \\
\hline EG & 8.73 & 9.09 & 8.97 & 9.26 & 9.5 & 9.2 & $9.13 \pm 0.26^{*}$ \\
\hline RG & 13.75 & 13.64 & 13.97 & 13.53 & 12.98 & 13.64 & $13.58 \pm 0.33$ \\
\hline \multicolumn{8}{|l|}{14} \\
\hline EG & 9.09 & 9.38 & 8.79 & 9.03 & 9.09 & 8.67 & $9.01 \pm 0.25^{*}$ \\
\hline RG & 14.19 & 12.76 & 13.09 & 14.19 & 13.75 & 12.98 & $13.49 \pm 0.63$ \\
\hline
\end{tabular}

Note. ${ }^{*} P<0.05$ versus an RG group. Stress values are positive (tensile) due to elongation caused by forward flexion load.

to avoid fixation failure [34]. Selective stress occlusion is another way to address stress-induced osteoporosis [35]. Vertebral compression stress stimulates bone growth and strengthens. Selective stress occlusion makes the internal fixation mainly against lateral bending, rotation and shear stress, reducing the compressive stress on internal fixation, thereby increasing the compressive stress of the vertebral body and compressive stress of vertebra. All these results can improve mechanical performance. Ti-24Nb-4Zr-8Sn fixation improved patient's postoperative symptoms significantly when compared with fusion surgery with Ti-6A1-4V [36].

Lumbar Ti-24Nb-4Zr-8Sn internal fixation system has the following advantages: retaining motion function of the 
TABLE 6: The stress resistance of Ti-24Nb-4Zr-8Sn and Ti-6A1-4V fixation groups under backward flexion loading after fixation (MPa).

\begin{tabular}{|c|c|c|c|c|c|c|c|}
\hline Measuring points & Sample 1 & Sample 2 & Sample 3 & Sample 4 & Sample 5 & Sample 6 & Mean \pm SD \\
\hline \multicolumn{8}{|l|}{1} \\
\hline EG & -4.7 & -4.88 & -4.78 & -4.93 & -5.01 & -4.95 & $-4.88 \pm 0.15^{*}$ \\
\hline RG & -5.95 & -5.86 & -6.12 & -5.84 & -6.11 & -5.78 & $-5.94 \pm 0.13$ \\
\hline \multicolumn{8}{|l|}{2} \\
\hline EG & -4.82 & -4.99 & -4.93 & -4.78 & -4.9 & -4.86 & $-4.88 \pm 0.07^{*}$ \\
\hline RG & -6.16 & -6.09 & -5.88 & -6.14 & -5.93 & -5.86 & $-6.01 \pm 0.14$ \\
\hline \multicolumn{8}{|l|}{3} \\
\hline EG & -2.09 & -2.44 & -2.5 & -2.32 & -2.48 & -2.36 & $-2.37 \pm 0.15^{*}$ \\
\hline RG & -1.77 & -1.84 & -1.71 & -1.73 & -1.69 & -1.75 & $-1.75 \pm 0.05$ \\
\hline \multicolumn{8}{|l|}{4} \\
\hline EG & -2.32 & -2.36 & -2.05 & -2.19 & -2.4 & -2.11 & $-2.24 \pm 0.14^{*}$ \\
\hline RG & -1.67 & -1.88 & -1.63 & -1.77 & -1.84 & -1.79 & $-1.76 \pm 0.10$ \\
\hline \multicolumn{8}{|l|}{5} \\
\hline EG & -4.99 & -4.88 & -4.93 & -4.78 & -5.03 & -4.9 & $-4.92 \pm 0.09^{*}$ \\
\hline RG & -6.03 & -6.16 & -6.09 & -5.86 & -6.16 & -6.11 & $-6.07 \pm 0.11$ \\
\hline \multicolumn{8}{|l|}{6} \\
\hline EG & -4.93 & -4.76 & -5.05 & -4.72 & -4.8 & -4.97 & $-4.87 \pm 0.13^{*}$ \\
\hline RG & -5.93 & -5.89 & -5.99 & -6.11 & -6.09 & -5.97 & $-6.00 \pm 0.09$ \\
\hline \multicolumn{8}{|l|}{7} \\
\hline EG & -2.38 & -2.19 & -2.52 & -2.09 & -2.36 & -2.34 & $-2.31 \pm 0.15^{*}$ \\
\hline RG & -1.67 & -1.61 & -1.82 & -1.86 & -1.69 & -1.79 & $-1.74 \pm 0.10$ \\
\hline \multicolumn{8}{|l|}{8} \\
\hline EG & -2.44 & -2.09 & -2.46 & -2.25 & -2.4 & -2.3 & $-2.32 \pm 0.14^{*}$ \\
\hline RG & -1.8 & -1.71 & -1.73 & -1.79 & -1.86 & -1.63 & $-1.75 \pm 0.08$ \\
\hline \multicolumn{8}{|l|}{9} \\
\hline EG & -1.67 & -1.59 & -1.75 & -1.57 & -1.69 & -1.73 & $-1.67 \pm 0.07^{*}$ \\
\hline RG & -1.88 & -2.25 & -2 & -2.25 & -1.98 & -2.28 & $-2.11 \pm 0.17$ \\
\hline \multicolumn{8}{|l|}{10} \\
\hline EG & -1.57 & -1.73 & -1.67 & -1.61 & -1.56 & -1.71 & $-1.64 \pm 0.07^{*}$ \\
\hline RG & -2.02 & -2.11 & -2.09 & -2.32 & -2.02 & -2.19 & $-2.13 \pm 0.11$ \\
\hline \multicolumn{8}{|l|}{11} \\
\hline EG & -11.74 & -11.56 & -12.57 & -12.33 & -12.39 & -12.21 & $-12.13 \pm 0.40^{*}$ \\
\hline RG & -15.95 & -16.61 & -17.05 & -16.5 & -17.93 & -17.38 & $-16.90 \pm 0.70$ \\
\hline \multicolumn{8}{|l|}{12} \\
\hline EG & -12.45 & -12.21 & -11.68 & -12.45 & -12.8 & -12.15 & $-12.29 \pm 0.38^{*}$ \\
\hline RG & -17.16 & -16.28 & -17.71 & -17.27 & -16.83 & -16.28 & $-16.92 \pm 0.57$ \\
\hline \multicolumn{8}{|l|}{13} \\
\hline EG & -11.62 & -13.04 & -12.33 & -12.74 & -12.04 & -12.86 & $-12.44 \pm 0.54^{*}$ \\
\hline RG & -16.83 & -16.17 & -17.82 & -16.17 & -18.15 & -16.94 & $-17.01 \pm 0.83$ \\
\hline \multicolumn{8}{|l|}{14} \\
\hline EG & -12.04 & -11.98 & -12.74 & -12.98 & -12.33 & -12.8 & $-12.48 \pm 0.42^{*}$ \\
\hline RG & -17.6 & -17.05 & -16.83 & -17.16 & -17.71 & -16.06 & $-17.07 \pm 0.60$ \\
\hline
\end{tabular}

Note. ${ }^{*} P<0.05$ versus an RG group. Stress values are negative due to compressive pressure caused by backward flexion load.

surgical segment while reducing the stress resistance of adjacent segments, delaying the occurrence of degeneration and even less damage to the disc, providing sufficient stability while retaining a certain degree of activity of lumbar spine, and delaying the occurrence of adjacent segment degeneration. This experiment is done by establishing a biomechanical model with Ti-24Nb-4Zr-8Sn fixation. The results showed that the effects of stress occlusion caused by Ti-24Nb-4Zr- $8 \mathrm{Sn}$ fixation were less than those caused by Ti-6A1-4V fixation.

Limitations of the Present Work. There were some limitations of the present study: Ti-24Nb-4Zr-8Sn fixation lacks longterm randomized controlled outcome, and long-term clinical stability, screws loosening, fatigue of screw, and connecting 
rods remain unclear; the specimens used for the lumbar spine may cause different results when compared with other specimens; only $\mathrm{Ti}-24 \mathrm{Nb}-4 \mathrm{Zr}-8 \mathrm{Sn}$ and $\mathrm{Ti}-6 \mathrm{Al}-4 \mathrm{~V}$ fixation were used to compare stress-shielding effects. In the posterior extension, compression, left and right bending, and stress need to be further analyzed in the future.

\section{Conclusion}

After fixation, strain and stress resistance of measurement points 1, 2, 5, 6, 9, and 10-14 in Ti-24Nb-4Zr-8Sn fixation was lower than that of Ti-6Al-4V fixation after forward and backward flexion loading. In contrast, the strain and stress resistance of measurement points 3, 4, 7, and 8 was higher in Ti-24Nb-4Zr-8Sn fixation than that of Ti-6Al-4V fixation. Stress-shielding effects of Ti-24Nb-4Zr-8Sn fixation were less than those of Ti-6Al-4V fixation. Ti-24Nb4Zr-8Sn fixation has more biomechanical properties than conventional Ti-6Al-4V fixation by reducing stress-shielding effects.

\section{Conflicts of Interest}

The authors have no conflicts of interest to disclose.

\section{References}

[1] C. Zhang, S. H. Berven, M. Fortin, and M. H. Weber, "Adjacent Segment Degeneration Versus Disease After Lumbar Spine Fusion for Degenerative Pathology: A Systematic Review With Meta-Analysis of the Literature," Clinical Spine Surgery, vol. 29, no. 1, pp. 21-29, 2016.

[2] S. Imagama, K. Ando, K. Kobayashi et al., "Atypical vertebral column fracture at the middle of fused area after instrumented posterior decompression and fusion surgery for beak type thoracic ossification of the posterior longitudinal ligament," Journal of Orthopaedic Science, 2016.

[3] T. Minato, M. Miyagi, W. Saito et al., "Spinal Epidural hematoma after thoracolumbar posterior fusion surgery without decompression for thoracic vertebral fracture," Case Reports in Orthopedics, Article ID 6295817, 2016.

[4] B. C. Kennedy, R. S. D’Amico, B. E. Youngerman et al., "Long-term growth and alignment after occipitocervical and atlantoaxial fusion with rigid internal fixation in young children," Journal of Neurosurgery: Pediatrics, vol. 17, no. 1, pp. 94102, 2016.

[5] T. C. Hankinson, A. M. Avellino, D. Harter et al., "Equivalence of fusion rates after rigid internal fixation of the occiput to C-2 with or without C-1 instrumentation: Clinical article," Journal of Neurosurgery: Pediatrics, vol. 5, no. 4, pp. 380-384, 2010.

[6] C. H. Lee, Y. E. Kim, H. J. Lee, D. G. Kim, and C. H. Kim, "Biomechanical effects of hybrid stabilization on the risk of proximal adjacent-segment degeneration following lumbar spinal fusion using an interspinous device or a pedicle screwbased dynamic fixator," Journal of Neurosurgery: Spine, pp. 1-7, 2017.

[7] B. Wang, Y. Fan, J. Dong et al., "A retrospective study comparing percutaneous and open pedicle screw fixation for thoracolumbar fractures with spinal injuries," Medicine (United States), vol. 96, no. 38, Article ID e8104, 2017.
[8] M. Aziz-Kerrzo, K. G. Conroy, A. M. Fenelon, S. T. Farrell, and C. B. Breslin, "Electrochemical studies on the stability and corrosion resistance of titanium-based implant materials," Biomaterials, vol. 22, no. 12, pp. 1531-1539, 2001.

[9] Y.-Y. Hsieh, C.-H. Chen, F.-Y. Tsuang, L.-C. Wu, S.-C. Lin, and C.-J. Chiang, "Removal of fixation construct could mitigate adjacent segment stress after lumbosacral fusion: A finite element analysis," Clinical Biomechanics, vol. 43, pp. 115-120, 2017.

[10] Y. Zhang, J.-L. Shan, X.-M. Liu, F. Li, K. Guan, and T.-S. Sun, "Comparison of the dynesys dynamic stabilization system and posterior lumbar interbody fusion for lumbar degenerative disease," PLoS ONE, vol. 11, no. 1, Article ID 0148071, 2016.

[11] C.-H. Lee, T.-A. Jahng, S.-J. Hyun et al., "Dynamic stabilization using the Dynesys system versus posterior lumbar interbody fusion for the treatment of degenerative lumbar spinal disease: A clinical and radiological outcomes-based meta-analysis," Neurosurgical Focus, vol. 40, no. 1, article no. E7, 2016.

[12] C. C. Ko, H. W. Tsai, W. C. Huang et al., "Screw loosening in the Dynesys stabilization system: radiographic evidence and effect on outcomes," Neurosurg Focus, vol. 28, no. 6, p. E10, 2010.

[13] M. C. Kennady, M. R. Tucker, G. E. Lester, and M. J. Buckley, "Stress shielding effect of rigid internal fixation plates on mandibular bone grafts. A photon absorption densitometry and quantitative computerized tomographic evaluation," International Journal of Oral and Maxillofacial Surgery, vol. 18, no. 5, pp. 307-310, 1989.

[14] Z.-X. Wu, C. Zhan, G. Cui et al., "Stress distribution on the screws in posterior lumbar fusion of isthmic spondylolisthesis with 2- or 3-vertebra fixation techniques: A biomechanical cadaveric study," Journal of Surgical Research, vol. 176, no. 1, pp. 95-101, 2012.

[15] Y.-B. Wang, L.-S. He, and L. Tian, "Effect of rigid internal fixation on mandibular growth in rabbits: experimental study," Journal of Traumatic Surgery, vol. 3, p. 017, 2011.

[16] D. Fukui, M. Kawakami, T. Matsumoto, and M. Naiki, "Stress enhances gait disturbance induced by lumbar disc degeneration in rat," European Spine Journal, pp. 1-9, 2017.

[17] J. Chen, J. Xuan, Y.-T. Gu et al., "Celastrol reduces IL-1 $\beta$ induced matrix catabolism, oxidative stress and inflammation in human nucleus pulposus cells and attenuates rat intervertebral disc degeneration in vivo," Biomedicine \& Pharmacotherapy, vol. 91, pp. 208-219, 2017.

[18] K. Schwartz, M. Rodrigo-Domingo, and T. Jensen, "Skeletal stability after large mandibular advancement $(>10 \mathrm{~mm})$ with bilateral sagittal split osteotomy and skeletal elastic intermaxillary fixation," Journal of Oral \& Maxillofacial Research, vol. 7, no. 2, p. e5, 2016.

[19] S. E. Doran, S. M. Papadopoulos, and L. D. Miller, "Internal fixation of the spine using a braided titanium cable: Clinical results and postoperative magnetic resonance imaging," Neurosurgery, vol. 38, no. 3, pp. 493-497, 1996.

[20] F. H. Geisler, S. E. Mirvis, H. Zrebeet, and J. Joslyn N; " Titanium wire internal fixation for stabilization of injury of the cervical spine: Clinical results and postoperative magnetic resonance imaging of the spinal cord," Neurosurgery, vol. 25, no. 3, pp. 356362, 1989.

[21] S. H. Lee, I. Chung, D. S. Choi et al., "Visual loss due to optic nerve infarction and central retinal artery occlusion after spine surgery in the prone position," Medicine (United States), vol. 96, no. 31, Article ID e7379, 2017. 
[22] M. Komatsu, K. Suda, M. Takahata et al., "Delayed bilateral vertebral artery occlusion after cervical spine injury: a case report," Spinal Cord Ser Cases, vol. 2, p. 16031, 2016.

[23] M. Benedetti Valentini, E. Ippolito, F. Catellani, and P. Farsetti, "Internal fixation after fracture or osteotomy of the femur in young children with polyostotic fibrous dysplasia," Journal of Pediatric Orthopaedics B, vol. 24, no. 4, pp. 291-295, 2015.

[24] K. C. Nune, R. D. K. Misra, S. J. Li, Y. L. Hao, and R. Yang, "Cellular response of osteoblasts to low modulus $\mathrm{Ti}-24 \mathrm{Nb}$ 4Zr-8Sn alloy mesh structure," Journal of Biomedical Materials Research Part A, vol. 105, no. 3, pp. 859-870, 2017.

[25] K. C. Nune, R. D. K. Misra, S. J. Li, Y. L. Hao, and R. Yang, "Osteoblast cellular activity on low elastic modulus $\mathrm{Ti}-24 \mathrm{Nb}-$ 4Zr-8Sn alloy," Dental Materials, vol. 33, no. 2, pp. 152-165, 2017.

[26] T. Mustafy, M. El-Rich, W. Mesfar, and K. Moglo, "Investigation of impact loading rate effects on the ligamentous cervical spinal load-partitioning using finite element model of functional spinal unit C2-C3," Journal of Biomechanics, vol. 47, no. 12, pp. 2891-2903, 2014.

[27] M. Brummund, V. Brailovski, Y. Petit, Y. Facchinello, and J. M. Mac-Thiong, "Impact of spinal rod stiffness on porcine lumbar biomechanics: Finite element model validation and parametric study," Proceedings of the Institution of Mechanical Engineers, Article ID 954411917732596, 2017.

[28] C.-H. Lee, P. R. Landham, R. Eastell, M. A. Adams, P. Dolan, and L. Yang, "Development and validation of a subject-specific finite element model of the functional spinal unit to predict vertebral strength," Proceedings of the Institution of Mechanical Engineers, Part H: Journal of Engineering in Medicine, vol. 231, no. 9, pp. 821-830, 2017.

[29] Y. L. Hao, S. J. Li, S. Y. Sun, C. Y. Zheng, and R. Yang, "Elastic deformation behaviour of Ti-24Nb-4Zr-7.9Sn for biomedical applications," Acta Biomaterialia, vol. 3, no. 2, pp. 277-286, 2007.

[30] J.-R. Cha, Y.-C. Kim, C. Jang, W.-K. Yoo, and J. H. Cui, "Pedicle screw fixation and posterior fusion for lumbar degenerative diseases: Effects on individual paraspinal muscles and lower back pain; A single-center, prospective study," BMC Musculoskeletal Disorders, vol. 17, no. 1, article no. 63, 2016.

[31] K. Takahashi, H. Ozawa, N. Sakamoto, Y. Minegishi, M. Sato, and E. Itoi, "Influence of intramedullary stress on cervical spondylotic myelopathy," Spinal Cord, vol. 51, no. 10, pp. 761764, 2013.

[32] Y.-L. Hao, S.-J. Li, and R. Yang, "Biomedical titanium alloys and their additive manufacturing," Rare Metals, vol. 35, no. 9, pp. 661-671, 2016.

[33] Y. L. Hao, Z. B. Zhang, S. J. Li, and R. Yang, "Microstructure and mechanical behavior of a Ti-24Nb-4Zr-8Sn alloy processed by warm swaging and warm rolling," Acta Materialia, vol. 60, no. 5, pp. 2169-2177, 2012.

[34] S. Amin Yavari, J. van der Stok, S. M. Ahmadi et al., "Mechanical analysis of a rodent segmental bone defect model: The effects of internal fixation and implant stiffness on load transfer," Journal of Biomechanics, vol. 47, no. 11, pp. 2700-2708, 2014.

[35] R. Langer and M. Langer, "Osseous changes in the foot bones in patients with arterial occlusion and simultaneous polyneuropathy (author's transl)," Rontgenblatter, vol. 34, no. 9, pp. 353-357, 1981.

[36] K. Sharman, P. Bazarnik, T. Brynk et al., "Enhancement in mechanical properties of a $\beta$-titanium alloy by high-pressure torsion," Journal of Materials Research and Technology, vol. 4, no. 1, pp. 79-83, 2015. 


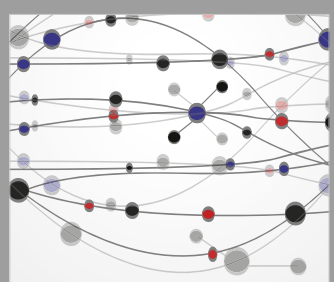

The Scientific World Journal
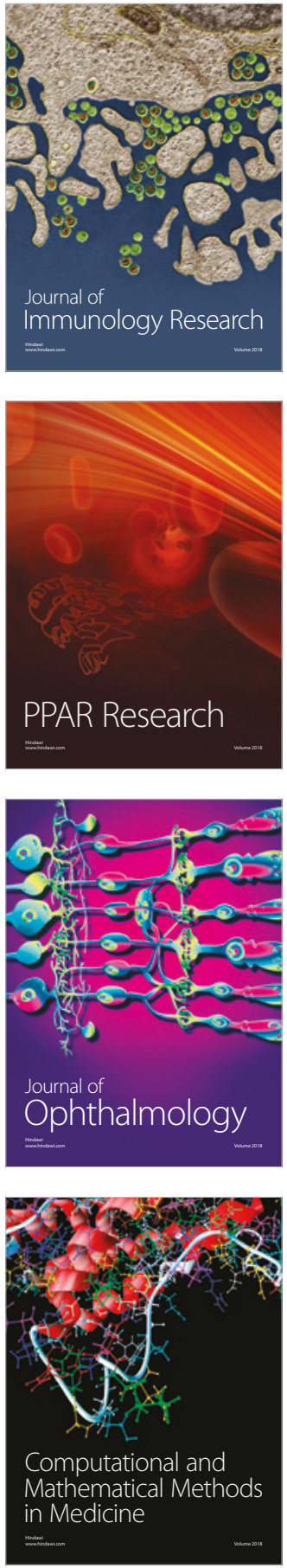

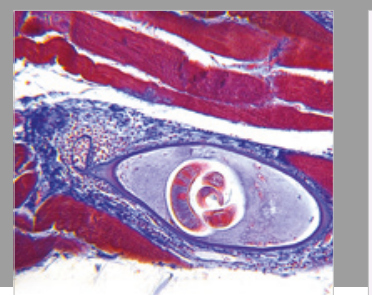

Gastroenterology Research and Practice

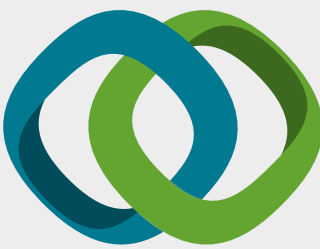

\section{Hindawi}

Submit your manuscripts at

www.hindawi.com
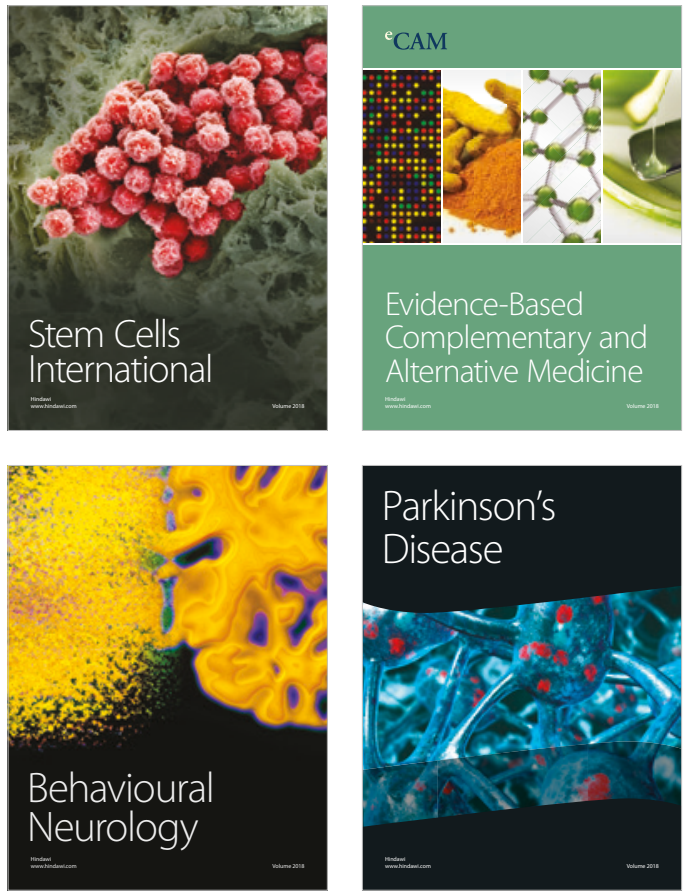

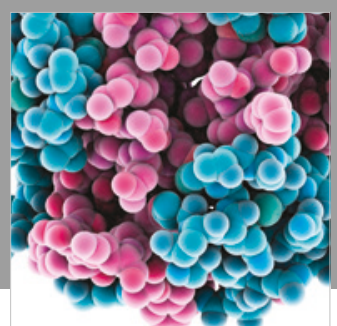

ournal of

Diabetes Research

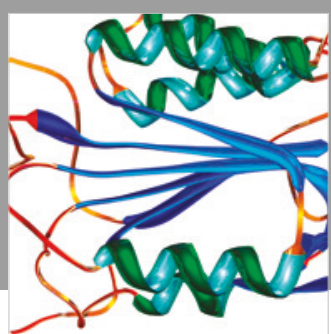

Disease Markers
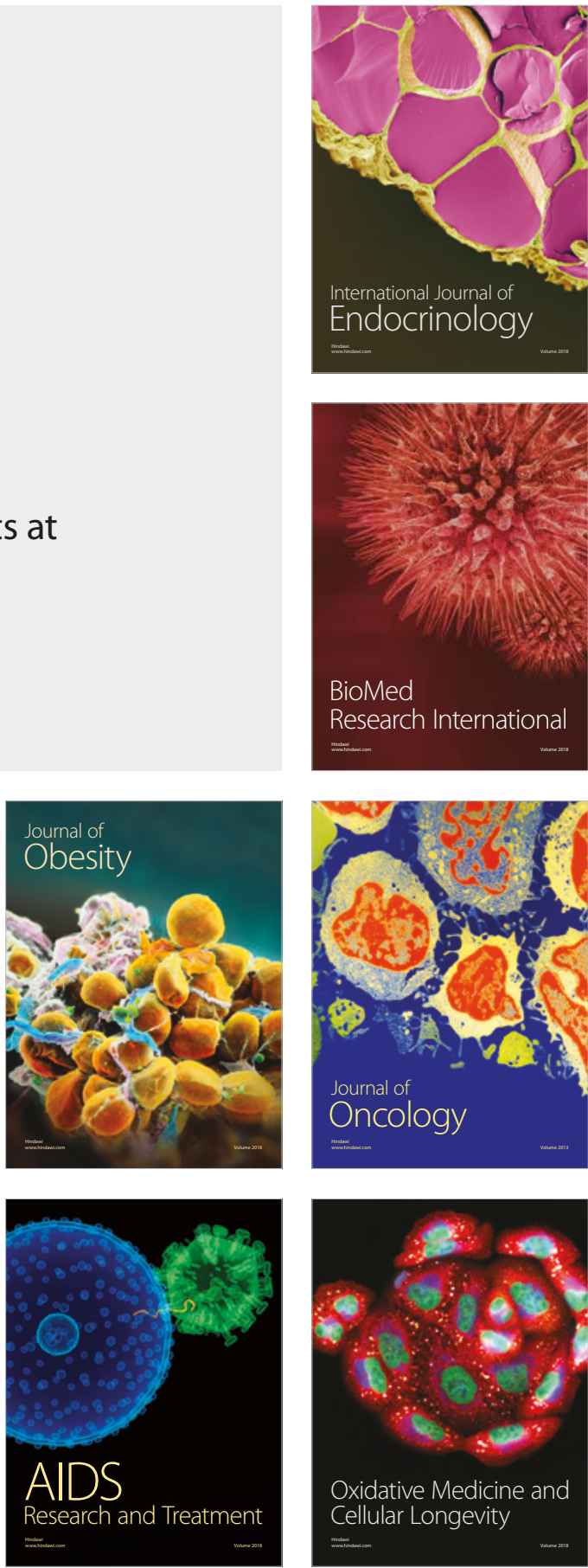